\title{
Acolhimento de imigrantes haitianos via integração ensino-serviço-pesquisa na atenção primária à saúde: relato de experiência
}

\author{
Accommodating Haitian immigrants via academia-service-research integration in primary \\ healthcare: case report
}
Acogida de inmigrantes haitianos vía integración enseñanza-servicio-investigación en la atención primaria de salud: relato de experiencia

Neoma Mendes Assis ${ }^{a}$, Letícia Lara Martinsª ${ }^{a}$ Lêda Maria Mendes Souzab ${ }^{b}$ Ivone Aparecida Nicolaoc ${ }^{c}$ Nathan Mendes Souzad

\section{Resumo}

Este artigo objetiva relatar a experiência do acolhimento a uma comunidade de imigrantes haitianos pela Atenção Primária à Saúde (APS) no âmbito do 'Internato de Atenção Integral à Saúde' do curso de Medicina da Unifenas - BH. A experiência ocorreu no Centro de Saúde Novo Aarão Reis (CSNAR) mediante estratégia multifacetada de acolhimento do serviço composta por: 1) interlocução com entidade de apoio a imigrantes, o Centro Zanmi;2) busca ativa e visitas domiciliares pela agente comunitária de saúde e internas de medicina, 3) aplicação de questionário; 4) apresentação dos resultados à comunidade de imigrantes haitianos, aos profissionais de saúde do CSNAR, à comunidade acadêmica da Unifenas - BH e a outros membros da comunidade interessados. Pelo contato com os primeiros haitianos identificados na comunidade, e por meio de busca ativa, outros foram encontrados e incluídos no estudo. Em conjunto com a aplicação dos questionários, foi possível avaliar o contexto em que estes se encontravam e, assim, buscar nas ferramentas da APS e do território formas de incluir esses imigrantes na comunidade e auxiliá-los em suas demandas. Este processo permitiu acolher a comunidade de imigrantes haitianos no CSNAR, otimizar seu cadastro no Sistema Único de Saúde, sensibilizar os profissionais de saúde para a importância do desenvolvimento de competência cultural e oportunizar intercâmbio de conhecimentos sobre o sistema de saúde haitiano e brasileiro.

\section{Abstract}

This article reports the experience of access by Haitian immigrants via academia-service-research integration in primary healthcare as part of the Unifenas - BH 'Internship on Primary Health Care'. The experience took place in the Primary Healthcare Centre Novo Aarão Reis (CSNAR) via a multifaceted accommodation service for the Haitian immigrant community comprised of: 1) dialogue with the immigrant support center; 2) active search and home visits by one community health agent and two medicine interns, 3) administration of questionnaires; 4) presentation of these research findings to the Haitian immigrant community, CSNAR health professionals, Unifenas - BH academic community, and other interested community members. This process enabled the accommodation of Haitian immigrants at CSNAR and their registration at the Unified Health System; sensitized health professionals to the importance of developing cultural competence and provided an opportunity to exchange knowledge about the Haitian and Brazilian health systems in a comparative perspective.

Como citar: Assis NM, Martins LL, Souza LMM, Nicolao IA, Souza NM. Acolhimento de imigrantes haitianos via integração ensino-serviço-pesquisa na atenção primária à saúde: relato de experiência. Rev Bras Med Fam Comunidade. 2017;12(39):1-9. http://dx.doi.org/10.5712/rbmfc12(39)1210
Palavras-chave: Atenção Primária à Saúde Acolhimento Acesso aos Serviços de Saúde Internato e Residência Emigrantes e Imigrantes Competência Cultural

Keywords: Primary Health Care User Embracement Health Services Accessibility Internship and Residency Emigrants and Immigrants Cultural Competency

Fonte de financiamento: declaram não haver. Parecer CEP: não se aplica. Conflito de interesses: declaram não haver. Procedência e revisão por pares: revisado por pares. Recebido em: 30/09/2016. Aprovado em: 08/05/2017. 


\section{Resumen}

Este artículo relata una experiencia de acceso a una comunidad de inmigrantes haitianos pela Atención Primaria a la Salud en el ámbito del "Internado de Atención Integral a la Salud" del Curso de Medicina de la Unifenas - BH. La experiencia ocurrió en el Centro de Salud Novo Aarão Reis (CSNAR) mediante la estrategia multifacética del servicio de acogida formado por: 1) diálogo con el centro de apoyo a los inmigrantes;2) búsqueda activa y visitas domiciliarias de las agentes comunitarias de salud y las internas de medicina, 3) administración de cuestionario; 4) presentación de los resultados a la comunidad inmigrante haitiana, a los profesionales de la salud del CSNAR, a la comunidad académica Unifenas - $\mathrm{BH}$ y a otros miembros de la comunidad interesados. Este proceso permitió acoger la comunidad de inmigrantes haitianos en el CSNAR, optimizar su registro en el Sistema Único de Salud, sensibilizar los profesionales de salud sobre la importancia del desarrollo de la competencia cultural y crear oportunidades para el intercambio de conocimientos sobre el sistema de salud de Haití y Brasil.
Palabras clave:

Atención Primaria a la Salud

Acogimiento

Accesibilidad a los

Servicios de Salud

Internado y Residencia

Emigrantes e Inmigrantes

Competencia Cultural

\section{Introdução}

O Sistema Único de Saúde (SUS) brasileiro tem seus fundamentos, diretrizes e atribuições encrustadas na Constituição da República Federativa do Brasil de 1988, via artigos que determinam os deveres do Estado e os direitos dos cidadãos. A garantia de igualdade de direitos a todos os brasileiros e estrangeiros, incluindo a proteção e assistência à saúde, consta nos artigos abaixo citados:

Art. 5o "Todos são iguais perante a lei, sem distinção de qualquer natureza, garantindo-se aos brasileiros e aos estrangeiros residentes no País a inviolabilidade do direito à vida, à liberdade, à igualdade, à segurança e à propriedade".

Art. 6o "São direitos sociais a educação, a saúde, a alimentação, o trabalho, a moradia, o lazer, a segurança, a previdência social, a proteção à maternidade e à infância, a assistência aos desamparados, na forma desta Constituição".

Art. 1960 "A saúde é direito de todos e dever do Estado, garantido mediante políticas sociais e econômicas que visem à redução do risco de doença e de outros agravos e ao acesso universal e igualitário às ações e serviços para sua promoção, proteção e recuperação".

O SUS, para garantir estes direitos, é constituído pelo conjunto das ações e de serviços de saúde sob gestão pública e suplementar. Organiza-se em redes regionalizadas e hierarquizadas em todo o território nacional, com direção única em cada esfera de governo. O SUS atua em consonância com as políticas públicas de seguridade social, que incluem Saúde, Previdência e Assistência Social. Assim, é dever do Estado, em cada ente da federação, prover acesso à saúde a brasileiros e estrangeiros, por meio dos princípios e atribuições do SUS, em todos os níveis de atenção. ${ }^{1-3}$

A Atenção Primária à Saúde (APS) é uma forma efetiva de organizar serviços de saúde guiada pela resolução das necessidades de saúde de uma determinada população. AAPS mantém consonância com as diretrizes do SUS respeitando a universalidade, integralidade, equidade e a corresponsabilidade entre população e governos. A APS é a porta de entrada ao sistema de saúde e o local responsável pela organização do cuidado integral e longitudinal à saúde dos indivíduos, suas famílias e da população. Tem capacidade para responder a cerca de $90 \%$ das necessidades em saúde, realizando serviços preventivos, curativos, reabilitadores e de promoção da saúde. 
AAPS também coordena os cuidados das pessoas no sistema de saúde com ações interdisciplinares, se necessário, e entre níveis secundários e terciários do SUS. ${ }^{4}$ Além destes atributos essenciais citados, a APS tem como atributos derivados: a orientação familiar, orientação comunitária e também a competência cultural dos profissionais. ${ }^{5,6}$

O curso de Medicina da Unifenas - campus de Belo Horizonte, está alicerçado no modelo educacional de aprendizagem baseada em problemas (PBL - problem based learning). Nasceu orientado às necessidades do SUS em conformidade com as Diretrizes Curriculares Nacionais (DCNs) do Curso de Graduação em Medicina (Resolução CNE/CES no 4 de 07/11/2001)7 e já se adequou às novas DCNs do Curso de Graduação em Medicina (Resolução CNE/CES n 3 de 20/06/2014) focando-se na formação de médicos generalistas. ${ }^{8}$

A proposta do Curso de Medicina da Unifenas - $\mathrm{BH}$ é formar profissionais preparados para suprir a crescente necessidade de médicos com perfil para atuação na APS. Desde o início do curso, os alunos treinam habilidades clínicas e atitudes médicas em cenário simulado, são inseridos no território das Unidades Básicas de Saúde (UBS) da rede de saúde de Belo Horizonte, onde realizam atividades, como diagnóstico situacional, promoção da saúde, e utilizam ferramentas de trabalho com famílias e comunidades.

No decorrer do curso, os alunos realizam atividades de atendimento clínico em ambiente ambulatorial, cujo objetivo é a prática da medicina centrada na pessoa. Nos dois anos finais do curso ocorre o Internato Médico Supervisionado, o qual mais de $30 \%$ é desenvolvido na APS e em Serviço de Urgência e Emergência do SUS atendendo às novas DCNs do Curso de Graduação em Medicina. ${ }^{7}$

Dada a integração ensino-serviço já consolidada no currículo da Unifenas - $\mathrm{BH}$, tenta-se avançar na construção de um sistema de saúde escola que também fomente a integração ensino-serviço à pesquisa. ${ }^{9-12}$

(...) os processos educativos possuem relevância não apenas por seus resultados - princípio da eficácia - mas porque são os responsáveis, entre outros aspectos, por criarem um ambiente intra e interpessoal favorável ao diálogo, à análise crítica das práticas, à reflexão, à construção de coletivos e à autonomização dos sujeitos facilitando e potencializando o processo de humanização da atenção e da gestão em saúde. ${ }^{11}$

Considerando o arcabouço legal do SUS, a implementação da APS como eixo estruturante do sistema, serviços e programas de saúde, os atributos essenciais e derivados da APS, o alinhamento curricular da Unifenas - BH com a nova DNC de 2014 e o compromisso do ensino inserido na realidade visando sua transformação, relata-se a experiência do acolhimento de imigrantes haitianos mediante uma estratégia multifacetada de integração ensino-serviço-pesquisa em Belo Horizonte, MG.

O contexto da imigração de haitianos para Belo Horizonte

O Haiti é um dos países com menor Índice de Desenvolvimento Humano (IDH) do mundo. Sua população vivencia há décadas graves problemas políticos e sociais e catástrofes naturais, como o terremoto ocorrido em janeiro de 2010, que matou milhares de pessoas, com ampla destruição de diversas cidades. No mesmo ano, um surto de cólera dizimou 8.000 pessoas no país. Em 2012, dois furacões, Issac e Sandy, atingiram o Haiti e comprometeram a produção agrícola do país. 
Essas sucessivas catástrofes contribuíram para a emergência de quadro de instabilidade política, econômica e social, que levaram a um efluxo de parcela considerável da população haitiana em busca de sobrevivência e de melhores condições de vida. O número estimado de emigrantes supera três milhões de pessoas, sendo os Estados Unidos, República Dominicana, Canadá, Cuba, Venezuela, e mais recentemente o Brasil, os principais destinos. ${ }^{13}$

A migração dos haitianos para o Brasil iniciou-se em 2010, quando somavam duas centenas, em 2011 estimou-se a presença de mais de 4.000 haitianos, em 2013 mais de 20.000 e em 2014 cerca de 50.000 haitianos no Brasil. ${ }^{14,15}$ Apesar das medidas governamentais e do apoio da sociedade civil organizada, a falta de instrumentos legais de uma política migratória adequada faz com que a chegada desses imigrantes ao país se transforme em uma situação única e desafiadora para toda a sociedade brasileira.

Cerca de $80 \%$ dos imigrantes haitianos no Brasil são do sexo masculino, estão entre 20 e 34 anos de idade e vieram ao Brasil em busca de trabalho, boa remuneração para auxiliar seus familiares que permanecem no Haiti e para estudo. Os primeiros haitianos que chegaram ao Brasil apresentavam maior qualificação profissional, diferentemente das imigrações recentes, constituída por número maior de pessoas com menor nível de instrução.

Os que conseguem obter o visto nas repartições consulares brasileiras seguem diretamente para a cidade de destino, partindo, na maioria dos casos, do Haiti, mas muitos conseguem o dinheiro da viagem com agiotas. As maiores dificuldades encontradas pelos imigrantes haitianos no Brasil são o idioma, baixos salários, habitação inadequada, baixo acesso a estudo e a dificuldade para validação de títulos haitianos de graduação e/ou profissionalizantes. ${ }^{13,14}$

Governos, organizações não governamentais e associações humanitárias têm-se empenhado no acolhimento aos imigrantes, via construção de abrigos, orientações para estabelecimento legal no país, acesso a benefícios sociais e emprego. Cidades da fronteira norte, Belo Horizonte, Curitiba, Porto Alegre, Rio de Janeiro e São Paulo foram escolhidas para acolhimento dos imigrantes devido à existência nestas cidades, e em municípios vizinhos, de um grande volume de haitianos que chegaram a estes destinos de várias formas. Belo Horizonte se transformou em um dos pontos de destino em destaque devido à presença de organizações humanitárias de apoio a refugiados ligadas à Igreja Católica, apoio da comunidade e também devido à facilidade para encontrar emprego na região. ${ }^{16,17}$

Os desafios da inclusão desses imigrantes refugiados relacionam-se ao idioma, à cultura diversa da brasileira, à situação de extrema vulnerabilidade social e requer da sociedade brasileira ações humanitárias e de solidariedade entre povos. Essa população precisa integrar-se às comunidades locais e, por isso, torna-se necessário que as cidades forneçam infraestrutura de acolhimento, informações e meios para regularização da estadia destes no Brasil. ${ }^{14}$

Os determinantes sociais da saúde (as condições sociais nas quais as pessoas vivem e trabalham) devem ser verificados na abordagem as pessoas e suas famílias no âmbito da APS. Tais determinantes refletem a posição ocupada pelas pessoas na hierarquia do status, do poder e do acesso aos recursos, auferindo pesada carga das doenças e iniquidades de saúde aos grupos populacionais em situação de desvantagem ou vulnerabilidade. ${ }^{18}$ As ações intersetoriais sobre estes determinantes estão consoantes com o modelo de APS abrangente proposta para a agenda do Saúde para Todos após a conferencia em Alma-Ata, em 1978, e a Estratégia Saúde da Família, defendida como modelo estruturante do SUS no Brasil. 


\section{Descrição e Discussão da Experiência}

Descrevemos neste relato a experiência de acolhimento de imigrantes haitianos no âmbito da APS pela equipe de saúde do CSNAR em parceria com a Unifenas - $\mathrm{BH}$ por meio de uma estratégia multifacetada de integração ensino-serviço-pesquisa em Belo Horizonte, MG.

A experiência se deu no no Centro de Saúde Novo Aarão Reis (CSNAR), pertencente à regional norte de Belo Horizonte. O CSNAR tem responsabilidade sanitária por cerca de 10.000 pessoas, a maioria oriunda do norte de Minas Gerais, do Vale do Jequitinhonha e também das regiões Norte e Nordeste do Brasil. Muitas destas famílias vivem em situação de elevado a muito elevado risco de vulnerabilidade social, daí a classificação da região como risco (C) pela Secretaria Municipal de Saúde. ${ }^{19}$

O Internato de Atenção Integral à Saúde (IAIS) no CSNAR propiciou intenso envolvimento e diálogo de saberes entre profissionais de saúde do CSNAR, acadêmicas da Unifenas - BH e a comunidade local. Esta integração ensino-serviço efetivou-se mediante identificação, planejamento e implementação de ação frente à preemente necessidade de provisão de serviços de saúde a comunidade de imigrantes haitianos localizada no território de abrangência do CSNAR.

A necessidade de abordagem à população de imigrantes haitianos foi identificada por uma Agente Comunitária de Saúde (ACS) do CSNAR, que vivia o desafio de abordar diversos haitianos de sua área de abrangência. Havia relato de dificuldade de acesso, provavelmente devido às barreiras linguística (crioulo/ francês x português) e geográfica (distância entre residência dos imigrantes e Centro de Saúde) e pela desconfiança dos imigrantes. O distanciamento entre a ACS e a situação de vulnerabilidade que muitos imigrantes haitianos viviam constituía importante demanda sentida pela equipe de saúde da família do CSNAR.

A experiência ocorreu em quatro fases: interlocução com o Centro Zanmi, associação de apoio a imigrantes de Belo Horizonte; busca ativa e visitas domiciliares pela ACS e internas de medicina; aplicação de questionário; apresentação dos resultados à comunidade de imigrantes haitianos, aos profissionais de saúde do CSNAR, à comunidade acadêmica da Unifenas - $\mathrm{BH}$ e a outros membros da comunidade interessados. Na fase de interlocução realizou-se visita à entidade de apoio a imigrantes, o Centro Zanmi, onde dialogamos com pessoas capacitadas no acolhimento a imigrantes, o que possibilitou informações importantes sobre abordagem a imigrantes, diferenças culturais e de linguagem.

A partir daí, iniciou-se a busca ativa de imigrantes haitianos por meio de visitas domiciliares (VD) realizadas pela ACS e as internas de medicina. Da abordagem no CSNAR quando algum dos imigrantes procurava por atendimento ou era levado por outros haitianos para ser apresentado à equipe. Esses o faziam após as VD, nas quais percebiam o interesse por parte da equipe de saúde em conhecer e auxiliar estas pessoas. Nestas VD e abordagens no CSNAR era aplicado um questionário individual aos haitianos residentes na área de abrangência do CSNAR, composto por um total de 30 questões, sendo 14 questões fechadas, 12 questões abertas, e quatro semiabertas.

Após a analise do questionário, verificamos tratar-se de pessoas jovens, com faixa etária predominante entre 25 e 35 anos, em sua maioria homens, casados e de baixa escolaridade. Sobre o motivo da vinda ao Brasil, apresentaram a mesma justificativa que foi encontrada na literatura, busca por trabalho, boa remuneração e uma vida melhor para seus familiares. A principal característica entre os casados foi que, 
após se estabelecerem, trouxeram seus cônjuges para também viver no Brasil. A população está, em média, há poucos meses no Brasil.

Os haitianos se vincularam ao bairro Novo Aarão Reis aparentemente sem motivo específico, alguns trazidos por contratantes, outros estimulados por familiares ou amigos que viviam em Belo Horizonte. Não identificaram dificuldades de adaptação ou deficiências na região, se adaptaram às condições de vida da comunidade local, apesar de manterem pouco convívio social com os moradores, sendo as igrejas o principal meio de lazer e convívio com os brasileiros. Estabeleceram-se em residências alugadas, a maioria vivendo com familiares em grupos de cerca de três pessoas e mantêm contato mensal com seus familiares no Haiti.

A interação do CSNAR com esta comunidade de imigrantes identificou uma frustação da mesma na medida em que a busca por melhores condições financeiras e qualidade de vida para si e para seus familiares no Haiti nem sempre foram alcançadas. Imigrantes com cursos superiores e profissões bem estabelecidas no Haiti tiveram que optar por atividades diferentes de suas formações profissionais. $O$ setor produtivo que mais emprega os homens é a construção civil e, para as mulheres, os serviços gerais. Trabalham, em geral, com carteira assinada e com carga horária semelhante à dos brasileiros.

Esta população possui duas crianças, uma nascida no Brasil há nove meses, com pré-natal realizado no CSNAR, e outra nascida no Haiti há quatro anos. Esta última encontra-se em idade escolar, porém está fora da escola por falta de vagas em creches do bairro. Identificou-se outra gestante também com acompanhamento pelo CSNAR, que relatou bom acesso e acompanhamento pré-natal pelo CSNAR. Esta gestante refere que os serviços de saúde superaram suas expectativas, por ter sido tratada com dignidade, experiência diferente a de outros países vividos anteriormente, onde relata ter sofrido racismo.

A maior parte da comunidade de haitianos do território refere uso anterior dos serviços do CSNAR, sendo que alguns também precisaram usar a atenção secundária. As demandas de saúde foram diversas e em sua maioria com resolução na APS. A principal dificuldade encontrada na adaptação dos haitianos ao SUS refere-se ao seu funcionamento, incluindo seus níveis assistenciais. Os entrevistados desconheciam os princípios do SUS (universalidade, integralidade, equidade e participação social) e o papel central da APS na rede de cuidados.

Os imigrantes relatam que no Haiti a assistência à saúde ocorre, majoritariamente, no hospital, centralizada no médico especialista focal e com financiamento privado. Consideram que o serviço público de saúde no Haiti tem acesso limitado e burocrático. Este desconhecimento levou alguns membros desta comunidade a recorrer ao sistema suplementar brasileiro. A distribuição de medicamentos no CSNAR e a quantidade de profissionais de saúde foram bem avaliados pela comunidade. Um ponto negativo do serviço do CSNAR foi a demora no atendimento e na realização de exames complementares.

Os imigrantes consideram positiva a distribuição gratuita de medicamentos pelo SUS, pois em seu país nativo não há esse benefício. Um ponto negativo do serviço do CSNAR foi a demora no atendimento e na realização de exames complementares. Quanto à queixa da demora no atendimento, a maioria se deu em relação aos pronto-atendimentos, pois baseado no modelo de saúde do Haiti a maioria se dirigia aos hospitais quando tinha alguma demanda de saúde, mesmo que de baixa complexidade. Após a intervenção da ACS e das internas de medicina, com elucidação do funcionamento do SUS, a maioria começou a utilizar mais o CSNAR e demonstrou maior satisfação em relação ao tempo de espera para o atendimento. 
Quando abordados sobre o atendimento do serviço social local, apenas uma haitiana referiu ter conhecimento e ter procurado o Centro de Referência de Assistência Social (CRAS). Esta imigrante haitiana possui situação de alta vulnerabilidade social, por isso foi referenciada pelo CSNAR ao CRAS. A população de imigrantes haitianos adscrita ao CSNAR praticamente desconhece o Centro Zanmi ${ }^{20}$ ou seus direitos e deveres como residentes no Brasil. ${ }^{18,21}$ Apenas um haitiano frequentou e participa de aulas de Português no Centro Zanmi.

Os haitianos acreditam que a baixa fluência em português e o baixo acesso a bons trabalhos sejam as maiores barreiras para o imigrante. Outras dificuldades estão relacionadas ao processo de adaptação cultural, relações de trabalho, baixa remuneração, custo de vida e distância dos familiares.

Para os haitianos, a vinda para o Brasil gerou grande expectativa, imaginavam grandes oportunidades de trabalho e renda. Apesar de não terem alcançado a expectativa em sua plenitude, avaliam que o Brasil oferece melhores condições de moradia, saúde, direitos e trabalho do que a que possuíam no Haiti. Seguem na busca por uma vida melhor e não hesitam em afirmar que pretendem voltar ao país de origem.

\section{Conclusão}

A realização deste relato de experiência é fruto da integração ensino-serviço-pesquisa entre o IAIS da Unifenas - BH, o Centro Zanmi e o CSNAR. Tal integração oportunizou o acolhimento das necessidades de saúde dos imigrantes haitianos residentes na área de abrangência do CSNAR mediante cadastramento no SUS e elucidação de seu funcionamento e suas diretrizes. Com isso, os haitianos puderam dirigir-se aos locais adequados de acordo com suas demandas; a vulnerabilidade socioeconômica em que se encontrava a maioria dos haitianos foi apresentada aos profissionais de saúde e do serviço social (CSNAR e CRAS, respectivamente), levando à sensibilização desses para esta população e facilitando o acesso dos haitianos a esses serviços, com auxílio de suas necessidades sociais, trabalhistas, jurídicas e de saúde; melhor acesso aos serviços públicos com explicação sobre a utilidade, localização e mobilidade aos mesmos; e o ganho de competência cultural de todos os profissionais envolvidos por meio do contato e intercâmbio com os haitianos, e da exposição dos resultados aos profissionais e internos da Unifenas - BH.

Foram identificadas as principais dificuldades de adaptação dos imigrantes residentes na área de abrangência do CSNAR, as demandas sociais e de saúde, além de possibilitar o conhecimento comparativo das diferenças no acesso e uso do sistema público de saúde brasileiro e haitiano. Ademais, estas ações contribuíram para a inserção da população de haitianos no contexto social belo-horizontino via contato direto com o Centro Zanmi, que a posteriori disponibilizou duas vagas para profissionais do CSNAR fazerem aulas de crioulo (dialeto haitiano).

Esta experiência também propiciou maior integração do CSNAR com demais setores da Rede de Assistência como a Gerência Regional, Gerência de Políticas de Igualdade Racial, que trabalham, desde então, com os profissionais do CSNAR para aprimorar a inserção, o resgate, a autoconfiança e o empoderamento da população negra haitiana no território, além de trazer à tona o olhar sobre os demais haitianos espalhados pela cidade de Belo Horizonte e ainda não visibilizados. 


\section{Referências}

1. Brasil. Constituição, 1988. Constituição da República Federativa do Brasil. Brasília: Senado Federal; 1988. [acesso 2015 Maio 9]. Disponível em: http://www.stf.jus.br/portal/constituicao

2. Andrade TMA. Da efetivação do direito à saúde no Brasil. 2010. [acesso 2015 Maio 9]. Disponível em: http://www.ambito-juridico.com. br/site/index.php?n_link=revista_artigos_leitura\&artigo_id=9037

3. Brasil. Conselho Nacional de Secretários de Saúde - CONASS. Sistema Único de Saúde/Conselho Nacional de Secretários de Saúde. [Internet] Brasília, 2011. 291 p. (Coleção Para Entender a Gestão do SUS 2011, 1). [acesso 2015 Maio 9]. Disponível em: http://www. conass.org.br/colecao2011/livro_1.pdf

4. Brasil. Conselho Nacional de Secretários de Saúde - CONASS. Atenção Primária e Promoção da Saúde. Coleção Progestores - Para entender a gestão do SUS. Brasília, 2007. p. 8-232. [acesso 2017 Maio 31]. Disponível em: http://bvsms.saude.gov.br/bvs/publicacoes/ colec_progestores_livro8.pdf

5. Lopes JMC. Princípios da Medicina de Família e Comunidade. In: Gusso G, Lopes JMC, orgs. Tratado de Medicina de Família e Comunidade. Princípios, Formação e Prática. Porto Alegre: Artmed; 2012. p. 1-11.

6. Takeda S. A Organização de Serviços de Atenção Primária à Saúde. In: Duncan BB, Schmidt MI, Giugliani ERJ, Duncan MS, Giugliani C. Medicina Ambulatorial. Condutas de Atenção Primária Baseadas em Evidências. Porto Alegre: Artmed; 2013. p. 19-32.

7. Universidade José do Rosário Vellano. Campus Belo Horizonte - UNIFENAS BH. [acesso 2015 Maio 2]. Disponível em: http://www. unifenas.br/medicinabh.asp?pasta=+BH-MED-11

8. Brasil. Ministério da Educação. Conselho Nacional de Educação. Resolução CNE/CES 3/2014, de 20 de junho de 2014 . Diretrizes curriculares nacionais dos cursos de graduação em medicina. Brasília: Ministério da Educação; 2014.

9. Frenk J. Reinventing primary health care: the need for systems integration. Lancet.2009;374(9684):170-3. DOI: http://dx.doi.org/10.1016/ S0140-6736(09)60693-0

10. Soares CHA, Pinto VPT, Dias MSA, Parente JRF, Chagas MIO. Sistema saúde escola de Sobral - CE. SANARE. 2008;7(2):7-13. [acesso 2015 Jun 1]. Disponível em: http://sanare.emnuvens.com.br/sanare/article/view/26

11. Dias MAS et al. Secretaria de Saúde de Sobral: construindo um sistema aprendente. Rev Sustentação. 2007;19:32-3.

12. Frenk J, Chen L, Bhutta ZA, Cohen J, Crisp N, Evans T, Fineberg H, et al. Health professionals for a new century: transforming education to strengthen health systems in an interdependent world. Lancet. 2010;376(9756):1923-58. DOI: http://dx.doi.org/10.1016/S01406736(10)61854-5

13. Assis GO. A “diáspora” haitiana a rumo ao Brasil e os desafios à política migratória brasileira: migrantes indesejados? Florianópolis: FAED - Universidade do Estado de Santa Catarina; 2012.

14. Costa GA. Haitianos em Manaus: dois anos de imigração - e agora! Travessia Rev Migrante; 2012;70:91-8.

15. Silva S. Brazil, a new Eldorado for immigrants? The case of Haitians and the Brazilian immigration policy. Urbanities. 2013;3(2):3-18.

16. Fernandes D. Projeto "Estudos sobre a Migração Haitiana ao Brasil e Diálogo Bilateral". Belo Horizonte; 2014. [acesso 2014 Dez 29]. Disponível em: http://obs.org.br/index.php?option=com_k2\&view=item\&id=746:projeto-estudos-sobre-a-migracaoXXXXXXXXXXahaitianahaitiana-ao-brasil-e-dialogo-bilateral\&Itemid=130

17. Fernandes D, Castro MCG, Ribeiro C. Migração Haitiana para o Brasil: Minas Gerais como destino, a fala dos haitianos. Belo Horizonte; 2014. [acesso 2017 Jun 1]. Disponível em: http://diamantina.cedeplar.ufmg.br/2014/site/arquivos/migracao-haitiana-para-o-brasil.pdf

18. Organização Mundial de Saúde (OMS). Ação sobre os determinantes sociais da saúde: aprendendo com experiências anteriores. Genebra: Organização Mundial de Saúde; 2005. [acesso 2015 Abr 15]. Disponível em: http://publicaciones.ops.org.ar/publicaciones/ piezas \%20comunicacionales/cursoDDS/cursoport/Textos\%20Completos/DSS\%20-\%20Aprendendo\%20com\%20experiencias\%20 anteriores.pdf 
19. Prefeitura de Belo Horizonte. Secretaria Municipal de Saúde. Projeto de Qualificação da Infraestrutura da Atenção Primária à Saúde de Belo Horizonte. Caracterização do Sistema Atual. Documento complementar 1. [acesso 2015 Set 15]. Disponível em: http://www.pbh. gov.br/smsa/atencao_primaria/Documento_Complementar_1G_Oeste.pdf

20. Centro Zanmi, do Serviço Jesuíta a Migrantes e Refugiados. [acesso 2015 Jan 20]. Disponível em: http://www.cpalsocial.org/110.html

21. Brasil. Ministério da Justiça. Agência da Organização das Nações Unidas (ONU) para refugiados. Alto comissariado das nações unidas para refugiados - ACNUR. Cartilha para refugiados no Brasil. [acesso 2015 Jan 20]. Disponível em: http://www.acnur.org/t3/fileadmin/ Documentos/portugues/Publicacoes/2015/Cartilha_para_solicitantes_de_refugio_no_Brasil_2015.pdf

a Universidade José do Rosário Vellano (UNIFENAS). Belo Horizonte, MG, Brasil. neomamendes@gmail.com; leticia_Imartins@hotmail.com

${ }^{\mathrm{b}}$ Internato de Atenção Integral à Saúde; Universidade José do Rosário Vellano (UNIFENAS). Belo Horizonte, MG, Brasil. ledamms@yahoo.com.br

`Centro de Saúde Novo Aarão Reis. Belo Horizonte, MG, Brasil. ivonendavid@yahoo.com.br a Universidade José do Rosário Vellano (UNIFENAS); Universidade Federal de Ouro Preto (UFOP). Belo Horizonte, MG, Brasil. nathanmendes@ @otmail.com (Autor correspondente) 